\title{
Longitudinal PET Monitoring of Amyloidosis and Microglial Activation in a Second-Generation Amyloid- $\beta$ Mouse Model
}

\author{
Christian Sacher*1, Tanja Blume*1,2, Leonie Beyer*1, Finn Peters ${ }^{2}$, Florian Eckenweber ${ }^{1}$, Carmelo Sgobio ${ }^{2}$, \\ Maximilian Deussing ${ }^{1}$, Nathalie L. Albert ${ }^{1}$, Marcus Unterrainer ${ }^{1}$, Simon Lindner ${ }^{1}$, Franz-Josef Gildehaus ${ }^{1}$, Barbara von \\ Ungern-Sternberg ${ }^{1}$, Irena Brzak ${ }^{3}$, Ulf Neumann ${ }^{3}$, Takashi Saito ${ }^{4}$, Takaomi C. Saido ${ }^{4}$, Peter Bartenstein ${ }^{1}$, \\ Axel Rominger ${ }^{1,5,6}$, Jochen Herms*2,5,7, and Matthias Brendel ${ }^{* 1,5}$ \\ ${ }^{I}$ Department of Nuclear Medicine, University Hospital of Munich, LMU Munich, Munich Germany; ${ }^{2} D Z N E-G e r m a n$ Center for \\ Neurodegenerative Diseases, Munich, Germany; ${ }^{3}$ Neuroscience, Novartis Institutes for BioMedical Research (NIBR), Basel, \\ Switzerland; ${ }^{4}$ Laboratory for Proteolytic Neuroscience, RIKEN Center for Brain Science, Saitama, Japan; ${ }^{5}$ Munich Cluster for \\ Systems Neurology (SyNergy), Munich, Germany; ${ }^{6}$ Department of Nuclear Medicine, Inselspital, University Hospital Bern, Bern, \\ Switzerland; and ${ }^{7}$ Center of Neuropathology and Prion Research, University of Munich, Munich, Germany
}

Nonphysiologic overexpression of amyloid- $\beta(A \beta)$ precursor protein in common transgenic $A \beta$ mouse models of Alzheimer disease likely hampers their translational potential. The novel $A p p^{N L-G-F}$ mouse incorporates a mutated knock-in, potentially presenting an improved model of Alzheimer disease for $A \beta$-targeting treatment trials. We aimed to establish serial small-animal PET of amyloidosis and neuroinflammation in $A p p^{N L-G-F}$ mice as a tool for therapy monitoring. Methods: $A p p^{N L-G-F}$ mice $(20$ homozygous and 21 heterogeneous) and 12 age-matched wild-type mice were investigated longitudinally from 2.5 to 10 mo of age with ${ }^{18} \mathrm{~F}$-florbetaben A PET and ${ }^{18} \mathrm{~F}-\mathrm{GE}-180$ 18-kDa translocator protein (TSPO) PET. Voxelwise analysis of SUV ratio images was performed using statistical parametric mapping. All mice underwent a Morris water maze test of spatial learning after their final scan. Quantification of fibrillar $A \beta$ and activated microglia by immunohistochemistry and biochemistry served for validation of the PET results. Results: The periaqueductal gray emerged as a suitable pseudo reference tissue for both tracers. Homozygous $A p p^{N L-G-F}$ mice had a rising SUV ratio in cortex and hippocampus for $A \beta(+9.1 \%,+3.8 \%)$ and TSPO $(+19.8 \%$, $+14.2 \%$ ) PET from 2.5 to 10 mo of age (all $P<0.05$ ), whereas heterozygous $A p p^{N L-G-F}$ mice did not show significant changes with age. Significant voxelwise clusters of $A \beta$ deposition and microglial activation in homozygous mice appeared at 5 mo of age. Immunohistochemical and biochemical findings correlated strongly with the PET data. Water maze escape latency was significantly elevated in homozygous $A p p^{N L-G-F}$ mice compared with wild-type at $10 \mathrm{mo}$ of age and was associated with high TSPO binding. Conclusion: Longitudinal PET in App NL-G-F knock-in mice enables monitoring of amyloidogenesis and neuroinflammation in homozygous mice but is insensitive to minor changes in heterozygous animals. The combination of PET with behavioral tasks in $A p p^{N L-G-F}$ treatment trials is poised to provide important insights in preclinical drug development.

Received Feb. 12, 2019; revision accepted May 15, 2019.

For correspondence or reprints contact: Matthias Brendel, Department of Nuclear Medicine, LMU Munich, Marchioninistrasse 15, Munich 81377 Germany.

E-mail: matthias.brendel@med.uni-muenchen.de

${ }^{*}$ Contributed equally to this work.

Published online Jul. 13, 2019.

COPYRIGHT (C 2019 by the Society of Nuclear Medicine and Molecular Imaging.
Key Words: Alzheimer disease; $\beta$-amyloid; microglia; $A p p^{N L-G-F}$; spatial learning

J Nucl Med 2019; 60:1787-1793

DOI: 10.2967/jnumed.119.227322

\section{A}

lzheimer disease (AD) is the most common neurodegenerative disease, with an incidence that increases exponentially with age, such that the prevalence exceeds $10 \%$ among octogenarians and $30 \%$ for nonagenarians. This epidemic is placing a growing socioeconomic burden on health care in societies with aging populations (1). The neuropathology of $\mathrm{AD}$ classically includes the accumulation of amyloid- $\beta$ peptide $(\mathrm{A} \beta)$ as extracellular plaques, and fibrillary tau aggregates within neurons. Activation of multiple neuroinflammatory pathways mediated by activated microglia expressing high levels of the marker $18-\mathrm{kDa}$ translocator protein (TSPO) completes the triad of markers. These pathologic conditions, restricted mainly to the cerebral cortex and the hippocampus, lead to a progressive decline in cognitive function, usually first manifesting as memory complaints (2-6). The identification of familial AD mutations in the amyloid precursor protein (APP) gene has led to the generation of several transgenic mouse models that overexpress APP $(7,8)$. These first-generation mouse models exhibit $\mathrm{AD}$ pathology, but the nonphysiologic overexpression of APP may cause additional phenotypes unrelated to AD. To circumvent these intrinsic drawbacks, second-generation APP knock-in mice that carry pathogenic mutations in the APP gene have been established (9). For example, $A p p^{N L-G-F}$ mice carry a mutant APP gene encoding the humanized $A \beta$ sequence (G601R, F606Y, and R609H) with 3 pathogenic mutations, namely Swedish (KM595/ 596NL), Beyreuther/Iberian (I641F), and Arctic (E618G). Homozygotic App ${ }^{N L-G-F}$ mice progressively exhibit widespread $\mathrm{A} \beta$ accumulation along with activation of microglia and astrocytes from 2 mo of age and express behavioral symptoms in the form of declining spatial learning ability from 8 to 12 mo of age (10-13). Given their physiologic expression of APP in comparison to transgenic mouse models, these knock-in mice are not characterized by massively elevated expression of the intracellular domain of APP or soluble APP $\alpha$ (9). Therefore, this mouse model potentially 
avoids confounds due to nonphysiologic signaling in therapy testing trials.

Previous studies have shown that small-animal PET is a suitable noninvasive tool for monitoring of therapeutic trials targeting AD pathology $(14,15)$. We previously established small-animal PET for monitoring of $A \beta$ deposition and microglial activation in APPoverexpressing mice, yielding excellent correlations with histologic and biochemical assessments (16). Given this background, the aim of this study was to transfer small-animal PET methodology to the $A p p^{N L-G-F}$ mouse model in a longitudinal investigation of the amyloid tracer ${ }^{18} \mathrm{~F}$-florbetaben and the TSPO tracer ${ }^{18} \mathrm{~F}-\mathrm{GE}$ 180. We confirmed the new dual-tracer PET results relative to findings obtained by immunohistochemistry and biochemistry and correlated the neuropathology findings with scores in a test of spatial learning.

\section{MATERIALS AND METHODS}

\section{Animals and Study Design}

All experiments were performed in compliance with the National Guidelines for Animal Protection, Germany, with the approval of the regional animal committee (Regierung Oberbayern) and were overseen by a veterinarian. Animals were housed in a temperature- and humidity-controlled environment with a 12-h light-dark cycle and free access to food (Sniff; Soest) and water. The experiments were performed in mixed-sex groups of heterozygous $(n=21)$ and homozygous $(n=20) A p p^{N L-G-F}$ mice, which is a knock-in mouse line generated by Saito et al. (11), and a group of age-matched wild-type mice. Small-animal PET examinations (A $\beta$ and TSPO) were performed in a longitudinal design at baseline ( $2.5 \mathrm{mo}$ of age $)$ and 3 follow-up measurements (5.0, 7.5, and $10.0 \mathrm{mo})$. Serial scans of both tracers deriving from a total of 12 age- and sex-matched wild-type mice served as controls, in consideration of the age-dependent increase of cortical TSPO PET signal in wild-type mice (17). All available mice underwent Morris water maze tests within $2 \mathrm{wk}$ after their scan. After behavioral testing, the mice were deeply anesthetized before transcardial perfusion and brain extraction. A minimum of 4 brains per genotype were processed for immunohistochemistry and biochemistry in randomly selected hemispheres.

\section{PET Imaging}

PET Data Acquisition, Reconstruction, and Postprocessing. For all PET procedures, we used an established standardized protocol for radiochemistry, acquisition, and preprocessing (16). In brief, ${ }^{18} \mathrm{~F}-\mathrm{GE}-$ 180 TSPO small-animal PET $(13.4 \pm 1.6 \mathrm{MBq} ; \sim 400-1,400 \mathrm{GBq} /$ $\mu \mathrm{mol})$ recordings with an emission window of 60-90 min after injection were obtained to measure cerebral TSPO expression, along with ${ }^{18} \mathrm{~F}$-florbetaben $\mathrm{A} \beta$-small-animal PET $(12.9 \pm 1.7 \mathrm{MBq} ; \sim 30-$ $80 \mathrm{GBq} / \mu \mathrm{mol}$ ) recordings with an emission window of 30-60 min after injection for assessment of fibrillar cerebral amyloidosis. Two $A p p^{N L-G-F}$ mice aged 11 mo were imaged in a dynamic setting $\left({ }^{18} \mathrm{~F}-\right.$ florbetaben: 0-60 $\mathrm{min}$ after injection; ${ }^{18} \mathrm{~F}-\mathrm{GE}-180$ : 0-90 $\mathrm{min}$ after injection) and their results compared with historic dynamic wild-type data for validation of the previously established time windows in this model. Anesthesia was maintained from just before tracer injection to the end of the imaging time window.

PET Image Analysis. We performed all analyses using PMOD (version 3.5; PMOD technologies). First, intensity normalization of images to SUV images was conducted by the previously validated myocardium correction method (18) for TSPO PET and by conventional SUV calculation for A $\beta$ PET. Voxel-based comparisons of SUV images between $A p p^{N L-G-F}(n=13$ per tracer, 10 mo) and wild-type mice ( $n=6$ per tracer, $10 \mathrm{mo}$ ) were performed to investigate a suitable pseudo reference tissue for PET quantification in the $A p p^{N L-G-F}$ mouse model. The judgment of suitability was also informed by the immunohistochemistry results described below. A suitable pseudo reference tissue was defined as a brain region lacking any genotypic difference in PET and immunohistochemistry results for both radioligands. These criteria led us to select the mesencephalic periaqueductal gray (PAG, comprising $20 \mathrm{~mm}^{3}$ ) as a pseudo reference region for calculation of SUV ratio (SUVR) for both $A \beta$ and TSPO PET (Fig. 1). Two bilateral frontal cortical target volumes of interest (VOIs, comprising $24 \mathrm{~mm}^{3}$ each) and 2 bilateral hippocampal target VOIs (comprising $10 \mathrm{~mm}^{3}$ each) were used for both tracers. Target-to-reference-tissue SUVRs were calculated for cortex (SUVR $\mathrm{CTX/PAG}_{\mathrm{P}}$ ) and hippocampus $\left(\mathrm{SUVR}_{\mathrm{HIP} / \mathrm{PAG}}\right)$ for $\mathrm{A} \beta$ and TSPO PET.

SPM Analysis. For both tracers, whole-brain voxelwise comparisons of PAG-scaled SUVR images between groups of knock-in and wild-type mice were performed as described previously $(19,20)$.

\section{Behavioral Testing}

Mice (homozygous App $p^{N L-G-F}: n=11$, heterozygous App $p^{N L-G-F}: n=$ 14, wild-type: $n=3$ ) underwent a Morris water maze test for spatial learning and memory deficits, which was performed according to a standard protocol with small adjustments (21). The video tracking software EthoVision XT (Noldus) was used for analyses of escape latency during the training period and during the probe trial.

\section{Immunohistochemistry and Biochemistry}

In brain regions corresponding to PET VOIs (Supplemental Table 1; supplemental materials are available at http://jnm.snmjournals.org), histochemistry was performed for fibrillar $\mathrm{A} \beta$ (methoxy-X04; Tocris) and immunohistochemistry for activated microglia using an Iba1 primary antibody (Wako) as previously established (17,22). NAB228 (Santa Cruz) was used for immunohistochemistry labeling of fibrillar

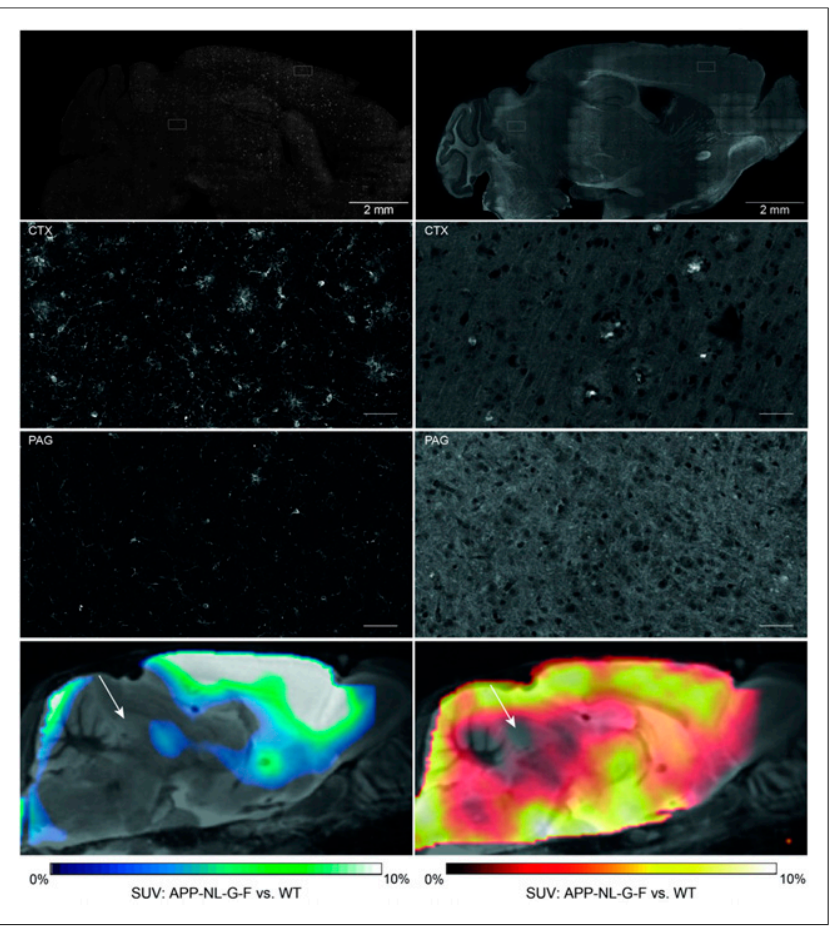

FIGURE 1. Immunohistochemistry reveals lowest microglial activation (left, lba-1) and amyloid deposition (right, methoxy-X04) in PAG of $A p p^{N L-G-F}$ mice aged 10 mo (overview and zoom in upper 3 panels). Suitability of PAG (white arrows) as pseudo reference tissue was further assessed by comparing SUV of TSPO- and A $\beta$ PET images between genotypes (overview in lowest panel). WT = wild-type. 
and nonfibrillar $\mathrm{A} \beta$ depositions. Hemispheres from 5 homozygous $A p p^{N L-G-F}, 5$ heterozygous $A p p^{N L-G-F}$, and 4 wild-type mice were used for immunohistochemistry. Assessment of $A \beta 40$ and $A \beta 42$ was performed as previously described (23). Biochemical analyses were performed in samples from the entire forebrain. Soluble Trem 2 protein was extracted from brain tissue with Tris-buffered saline, and measured by ELISA, using polyclonal sheep antibody for coating (AF1729; R\&D Systems) and biotinylated polyclonal sheep antibody (BAF1729; R\&D Systems) together with streptavidin-horseradish peroxidase (N-100; ThermoFisher Scientific) for detection. Hemispheres from 8 homozygous $A p p^{N L-G-F}, 14$ heterozygous $A p p^{N L-G-F}$, and 4 wild-type mice were used for biochemical analyses.

\section{Statistics}

Group comparisons of VOI-based PET results between knock-in and wild-type mice were performed by 1-way ANOVA and Tukey post hoc tests for multiple comparisons, calculated by SPSS 25 Statistics (IBM). Two-sided $t$ tests were used to compare terminal multimodal readouts of homozygous $A p p^{N L-G-F}$ with wild-type or heterozygous $A p p^{N L-G-F}$ groups. Two-way ANOVA was applied to assess methoxyX04 and NAB228 fluorescence intensity changes distant and close to plaques. For correlation analyses in $A p p^{N L-G-F}$, Pearson coefficients of correlation $(R)$ were calculated for normally distributed readouts after Kolmogorov-Smirnov testing for normalcy. For nonnormally distributed readouts, Spearman coefficients of correlation $\left(\mathrm{r}_{\mathrm{S}}\right)$ were calculated. A threshold $P$ value of less than 0.05 was considered significant for rejection of the null hypothesis. Sample size calculations for potential upcoming treatment trials were performed for longitudinal (2.5-10.0 mo) and terminal measures in the cortical VOI for both ligands in homozygous $A p p^{N L-G-F}$ mice. We used a simplified $t$-statistic model with assumptions of a type I error $\alpha$ of 0.05 , a power of 0.8 , and a treatment effect of $50 \%$ calculated in G*Power (version 3.1; Heinrich-Heine University). For the power calculation, we simulated the treatment group by calculating longitudinal differences within single $A p p^{N L-G-F}$ mice and terminal differences of single $A p p^{N L-G-F}$ mice by multiplying the mean endpoint of wild-type for each tracer by 0.5 , corresponding to the $50 \%$ treatment effect.

\section{RESULTS}

\section{Pseudo Reference Region}

Immunohistochemistry revealed a widespread amyloidosis and microglial activation in $A p p^{N L-G-F}$ mice at 10 mo of age, involving most regions of the forebrain (Fig. 1). Regions with relatively low amyloidosis and microglial activation were observed in parts of the hindbrain, that is, vermis, midbrain, and notably the PAG. SUV differences between genotypes at 10 mo of age fitted to immunohistochemistry and revealed the lowest ${ }^{18} \mathrm{~F}$-florbetaben and ${ }^{18} \mathrm{~F}-\mathrm{GE} 180$ alterations in the hindbrain (Fig. 1). SUV analysis at the final time point revealed that an oval VOI primarily comprising PAG voxels yields a suitable pseudo reference region $\left({ }^{18} \mathrm{~F}\right.$-florbetaben SUV: $A p p^{N L-G-F}, 0.47 \pm 0.08$; wild-type, $0.46 \pm 0.09$ [not statistically significant $] /{ }^{18} \mathrm{~F}-\mathrm{GE} 180 \mathrm{SUV}$ with myocardium correction (18): $A p p^{N L-G-F}$, $0.22 \pm 0.02$; wild-type, $0.23 \pm 0.02$ [not statistically significant]). SUVR $_{\text {CTX/PAG time-activity-curves of aged } A p p^{N L-G-F} \text { mice revealed }}$ stable uptake differences for 30-60 min after injection for ${ }^{18} \mathrm{~F}$-florbetaben and 60-90 min after injection for ${ }^{18} \mathrm{~F}-\mathrm{GE} 180$ imaging when compared with historic wild-type data (Supplemental Fig. 1). Furthermore, the comparison of methoxy-X04 and NAB228 staining revealed only a minor fraction of fibrillar $A \beta$ in amyloid plaques in the entire brain (Fig. 2), which predicted a relatively lower ${ }^{18} \mathrm{~F}$-florbetaben signal than in the historically investigated amyloid mouse models.

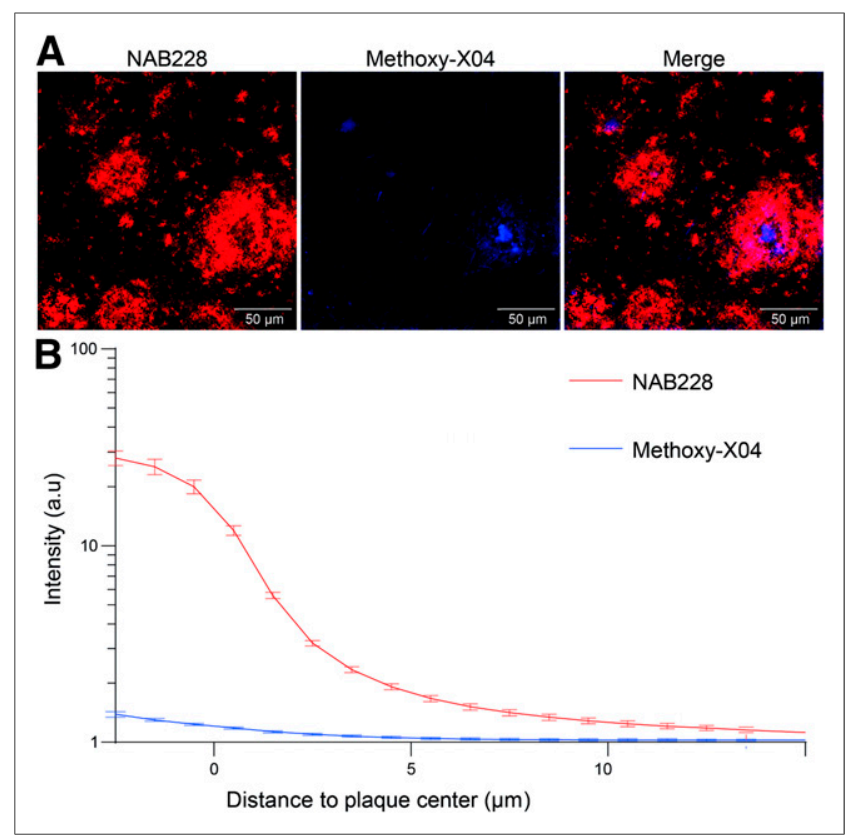

FIGURE 2. (A) Minor dense fraction of cortical $A \beta$ plaques in $A p p^{N L-G-F}$ mice as assessed by NAB228 (red) and methoxy-X04 (blue) costaining. (B) Graph indicates mean methoxy-X04 and NAB228 fluorescence intensity profiles from plaque border; 2-way ANOVA interaction staining $\times$ distance, $F_{43,704}=14.79, P<0.001$. Data are presented as mean \pm SEM with 9 mice per group. Minimal plaque number analyzed per mouse is $41 .{ }^{* \star \star} P<0.001$. a.u $=$ arbitrary units.

\section{Dual-Tracer PET Analyses}

A comprehensive overview of the PET results is provided in Table 1. The age dependence of the retention of the 2 tracers is presented in Figure 3 and illustrated in Supplemental Figure 2.

$A \beta$ PET Findings. Homozygous $A p p^{N L-G-F}$ mice already showed elevated cortical ${ }^{18} \mathrm{~F}$-florbetaben SUVR compared with their baseline as early as 5 mo of age $(+3.4 \% ; P<0.05)$, which increased further at $10 \mathrm{mo}(+9.1 \% ; P<0.001)$. Hippocampal increases in SUVR first became apparent at $7.5 \mathrm{mo}(+2.6 \% ; P<0.05)$ and were more conspicuous at $10 \mathrm{mo}(+3.8 \% ; P<0.001)$. Required sample sizes for detection of a $50 \% \mathrm{~A} \beta$ PET treatment effect in the cortex of homozygous $A p p^{N L-G-F}$ mice were $n=11$ for evaluation of longitudinal measures between 2.5 and 10 mo and $n=8$ for the terminal time point. The heterozygous genotype did not show significant changes in ${ }^{18} \mathrm{~F}$-florbetaben SUVR relative to baseline at any age.

TSPO PET Findings. Homozygous App ${ }^{N L-G-F}$ mice revealed the first evidence of increased cortical ${ }^{18} \mathrm{~F}-\mathrm{GE}-180$ uptake compared with baseline as early as $5 \mathrm{mo}(+6.5 \%$; $P<0.05)$, which increased strongly by 10 mo $(+19.8 \% ; P<0.001)$. Significantly elevated ${ }^{18} \mathrm{~F}$-GE-180 SUVR in the hippocampus was present at 7.5 mo $(+10.8 \% ; P<0.001)$, which increased further by 10 mo $(+14.2 \% ; P<0.001)$. Required sample sizes for detection of a $50 \%$ TSPO PET treatment effect in the cortex of homozygous $A p p^{N L-G-F}$ mice were $n=16$ for evaluation of longitudinal measures between 2.5 and 10 mo and $n=11$ for the terminal time point. The heterozygous genotype revealed neither cortical nor hippocampal microglial activation at any age.

Correlation Analyses. Significant positive associations between $\mathrm{A} \beta$ and TSPO PET quantification were observed for the cortex $(R=0.64 ; P<0.001$; Fig. $3 \mathrm{C})$ and the hippocampus $(R=0.48$; $P<0.05$; Fig. 3F). 
TABLE 1

Overview of Small-Animal PET Results

\begin{tabular}{|c|c|c|c|c|c|c|c|c|c|}
\hline \multirow[b]{3}{*}{ Group } & \multirow[b]{3}{*}{ Age (mo) } & \multicolumn{4}{|c|}{ Amyloid PET } & \multicolumn{4}{|c|}{ TSPO PET } \\
\hline & & \multirow[b]{2}{*}{$n$} & \multirow[b]{2}{*}{ Sex } & \multicolumn{2}{|c|}{ SUVR } & \multirow[b]{2}{*}{$n$} & \multirow[b]{2}{*}{ Sex } & \multicolumn{2}{|c|}{ SUVR } \\
\hline & & & & Cortex & Hippocampus & & & Cortex & Hippocampus \\
\hline \multirow[t]{4}{*}{ Homozygous App ${ }^{\text {NL-G-F }}$} & 2.5 & 20 & $90^{3} / 11$ q & $0.86 \pm 0.02$ & $0.95 \pm 0.01$ & 18 & $90^{x} / 90$ & $0.79 \pm 0.05$ & $0.82 \pm 0.04$ \\
\hline & 5.0 & 17 & $60^{3} / 11$ \% & $0.89 \pm 0.03^{*}$ & $0.96 \pm 0.02$ & 17 & $60^{7} / 11$ Q & $0.84 \pm 0.04^{*}$ & $0.86 \pm 0.03$ \\
\hline & 7.5 & 13 & $60^{3} / 7 \%$ & $0.92 \pm 0.05^{\dagger}$ & $0.97 \pm 0.03^{\star}$ & 14 & $60^{7} / 8 \%$ & $0.91 \pm 0.04^{\dagger}$ & $0.91 \pm 0.06^{\dagger}$ \\
\hline & 10.0 & 13 & $60^{x} / 79$ & $0.94 \pm 0.03^{\dagger}$ & $0.98 \pm 0.02^{\dagger}$ & 13 & $60^{x} / 79$ & $0.94 \pm 0.06^{\dagger}$ & $0.94 \pm 0.07^{\dagger}$ \\
\hline \multirow[t]{4}{*}{ Heterozygous App NL-G-F } & 2.5 & 21 & $130^{2} / 8 \%$ & $0.87 \pm 0.03$ & $0.95 \pm 0.03$ & 20 & $120^{\pi} / 8$ ? & $0.78 \pm 0.06$ & $0.81 \pm 0.04$ \\
\hline & 5.0 & 20 & $120^{x} / 8 \%$ & $0.87 \pm 0.04$ & $0.94 \pm 0.02$ & 20 & $120^{x} / 8 \%$ & $0.78 \pm 0.05$ & $0.81 \pm 0.04$ \\
\hline & 7.5 & 15 & $90^{n} / 6 ?$ & $0.89 \pm 0.04$ & $0.95 \pm 0.02$ & 17 & $100^{3} / 7 \%$ & $0.77 \pm 0.04$ & $0.81 \pm 0.05$ \\
\hline & 10.0 & 13 & $80^{x} / 59$ & $0.89 \pm 0.04$ & $0.95 \pm 0.03$ & 13 & $80^{n} / 59$ & $0.79 \pm 0.04$ & $0.81 \pm 0.05$ \\
\hline \multirow[t]{2}{*}{ Wild-type C57BL/6 } & 2.5 & 6 & $30^{x} / 39$ & $0.87 \pm 0.03$ & $0.96 \pm 0.01$ & 6 & $30^{x} / 39$ & $0.75 \pm 0.07$ & $0.80 \pm 0.04$ \\
\hline & 10.0 & 6 & $30^{x} / 30$ & $0.86 \pm 0.01$ & $0.95 \pm 0.01$ & 6 & $30^{x} / 30$ & $0.82 \pm 0.04$ & $0.84 \pm 0.03$ \\
\hline
\end{tabular}

${ }^{*} P<0.05$ (1-way ANOVA including post hoc Tukey testing vs. baseline).

${ }^{\dagger} P<0.001$ (1-way ANOVA including post hoc Tukey testing vs. baseline).

Numbers $(n)$ of mice included in PET analyses by sex are provided for each tracer and age.

Voxelwise Analyses. Voxelwise group contrasts between knockin and wild-type animals are shown in Supplemental Figure 3. By this exploratory approach, the strongest differences in ${ }^{18} \mathrm{~F}-$ florbetaben uptake between homozygous $A p p^{N L-G-F}$ and wild-type mice $(P<0.001$, uncorrected) were discerned in the left thalamus. This first became apparent at 5 mo, whereas comparable thalamic
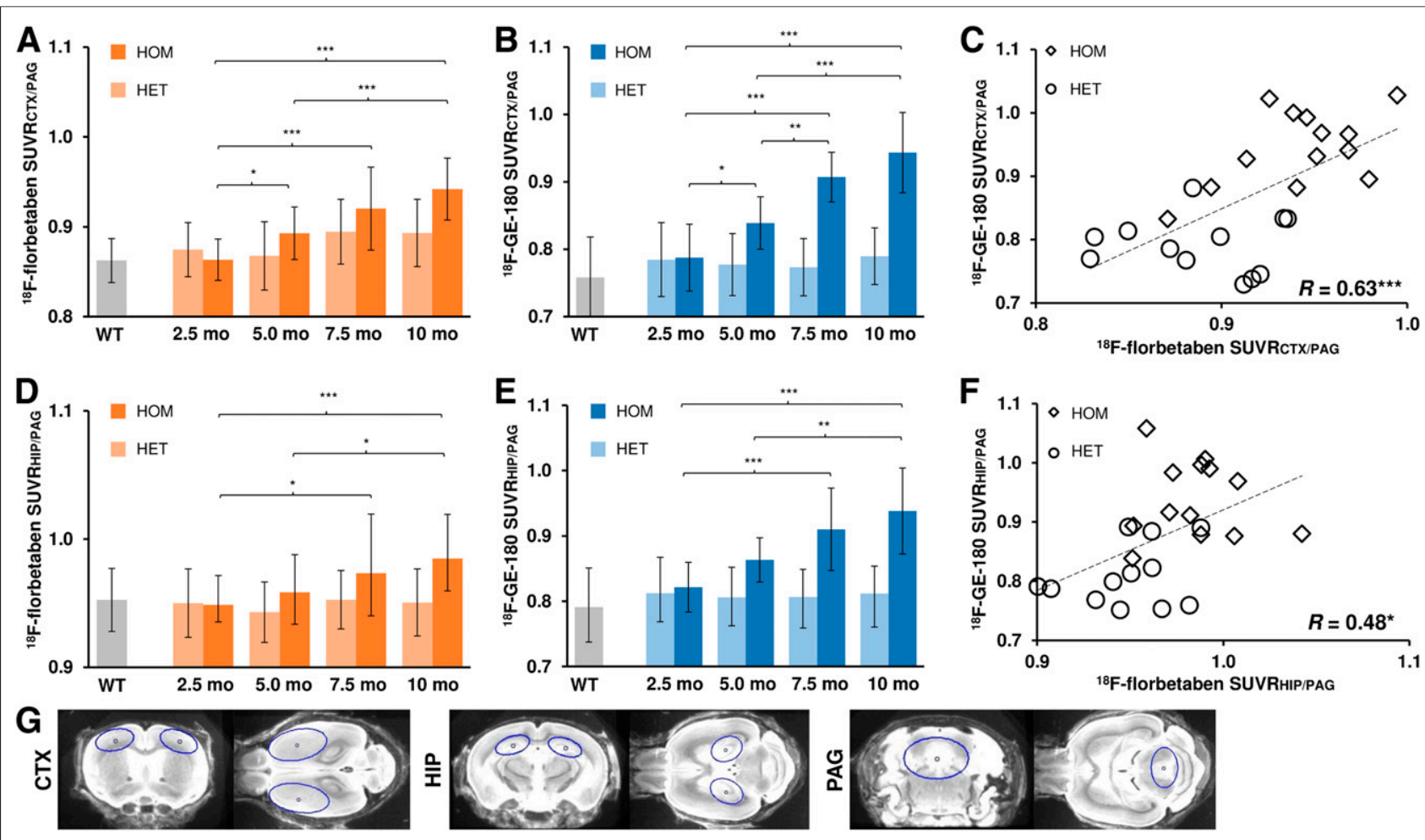

FIGURE 3. ( $A, B, D$, and $E)$ Age dependence of $A \beta$ and TSPO radiotracer uptake in frontal cortex and hippocampus of homozygous (HOM) and heterozygous (HET) App ${ }^{N L-G-F}$ mice. Group comparisons of VOI-based small-animal PET results between knock-in mouse groups were assessed by 1-way ANOVA and Tukey post hoc test. ( $\mathrm{C}$ and $\mathrm{F}$ ) Correlation between $\mathrm{A} \beta$ deposition and microglial activation in frontal cortex and in hippocampus measured by dual-tracer small-animal PET ( $R$ indicates Pearson coefficients of correlation). (G) Definitions of cortical (CTX), hippocampal (HIP), and PAG VOls in coronal and axial slices on MRI mouse brain atlas. ${ }^{\star} P<0.05 .{ }^{\star \star} P<0.01$. ${ }^{\star \star \star} P<0.001$. WT $=$ wild-type. 
elevations in the heterozygous genotype emerged only at $7.5 \mathrm{mo}$. Significant SUVR increases in neocortical areas were observed in homozygous $A p p^{N L-G-F}$ mice only at 10 mo of age, when $29 \%$ of the total brain volume had elevated ${ }^{18} \mathrm{~F}$-florbetaben signal relative to the wild-type group.

Voxelwise TSPO PET analysis revealed microglial activation in homozygous $A p p^{N L-G-F}$ mice in the frontal cortex and the hippocampus starting at 5 mo (18\% of total brain volume, $P<0.001$, uncorrected), which increased to involvement of $48 \%$ of total brain volume at 10 mo of age $(P<0.001$, uncorrected), including thalamic regions. Heterozygous $A p p^{N L-G-F}$ animals showed no differences in ${ }^{18} \mathrm{~F}-\mathrm{GE}-180$ uptake relative to wild-type at any age.

\section{Correlation with Multimodal Terminal Readouts}

Average values for the different genotypes of all terminal readouts at the age of 10 mo are presented in Supplemental Table 1. We observed strong increases in all biochemical (A $\beta 40, A \beta 42$, sTrem2) and (immuno)histochemistry (Iba1, methoxy-X04; Supplemental Fig. 4) readouts in the comparison of homozygous $A p p^{N L-G-F}$ with wild-type or heterozygous $A p p^{N L-G-F}$ animals. Spatial learning score was substantially impaired in the homozygous $A p p^{N L-G-F}$ compared with wild-type groups (latency to platform, +2.1-fold, $P<0.05$, 2-tailed), with no such difference for heterozygous $A p p^{N L-G-F}$. All correlations between SUVRs at 10 mo of age and multimodal terminal readouts are illustrated in Figure 4.

Biochemistry. A $\beta 42$ concentration correlated highly with cortical ${ }^{18}$ F-florbetaben $\left(\mathrm{r}_{\mathrm{S}}=0.69 ; P<0.001\right)$ and ${ }^{18} \mathrm{~F}-\mathrm{GE}-180$ uptake $\left(\mathrm{r}_{\mathrm{S}}=0.70 ; P<0.001\right)$. Furthermore, significant $\mathrm{A} \beta 42$ correlations with hippocampal SUVRs were observed for both tracers $(P<0.01)$. Quantification of sTrem2 correlated with the cortical $\left(\mathrm{r}_{\mathrm{S}}=0.61 ; P<0.01\right)$ and hippocampal $\left(\mathrm{r}_{\mathrm{S}}=0.53 ; P<0.05\right)$ SUVR of ${ }^{18} \mathrm{~F}-\mathrm{GE}-180$.
Immunohistochemistry. Hippocampal $\left(\mathrm{r}_{\mathrm{S}}=0.90 ; P<0.001\right)$ and cortical $(R=0.75 ; P<0.05){ }^{18} \mathrm{~F}$-florbetaben uptake strongly correlated with plaque burden, measured by methoxy-X04 histology in the corresponding regions. The Iba1 burden, which is indicative of activated microglia, correlated with uptake of the TSPO tracer ${ }^{18} \mathrm{~F}-\mathrm{GE}-180$ in neocortex $(R=0.92 ; P<0.001)$ and hippocampus $(R=0.78 ; P<0.01)$.

Behavioral Analysis. There was a moderate significant association between cortical ${ }^{18} \mathrm{~F}-\mathrm{GE}-180$ SUVR and escape latency at 10 mo $(R=0.41 ; P<0.05)$, meaning that mice with stronger microglial activation needed significantly more time to reach the platform in the Morris water maze test.

\section{DISCUSSION}

To our knowledge, this was the first longitudinal dual-tracer PET study of cerebral amyloidosis and neuroinflammation in a knock-in AD mouse model. After modification of standardized PET protocols to circumvent model-specific difficulties in homozygous $A p p^{N L-G-F}$ knock-in mice, we detected strong progressive increases of ${ }^{18} \mathrm{~F}$-florbetaben and ${ }^{18} \mathrm{~F}-\mathrm{GE}-180$ uptake with age. Terminal validation analyses by immunohistochemistry and biochemistry confirmed these in vivo PET results. The present findings establish the basis for serial PET monitoring of therapeutic agents targeting $\mathrm{A} \beta$ deposition and microglial activation in $A p p^{N L-G-F}$ mice.

Two model-specific issues were encountered and solved for establishing PET imaging in $A p p^{N L-G-F}$ mice: first, the widespread amyloid pathology in the brain hampered the use of previously established reference regions such as the cerebellum or white matter (16). SUVR scaling by an appropriate intracerebral reference tissue represents an important tool to generate robust PET results during short acquisitions in mice. This is crucial for the

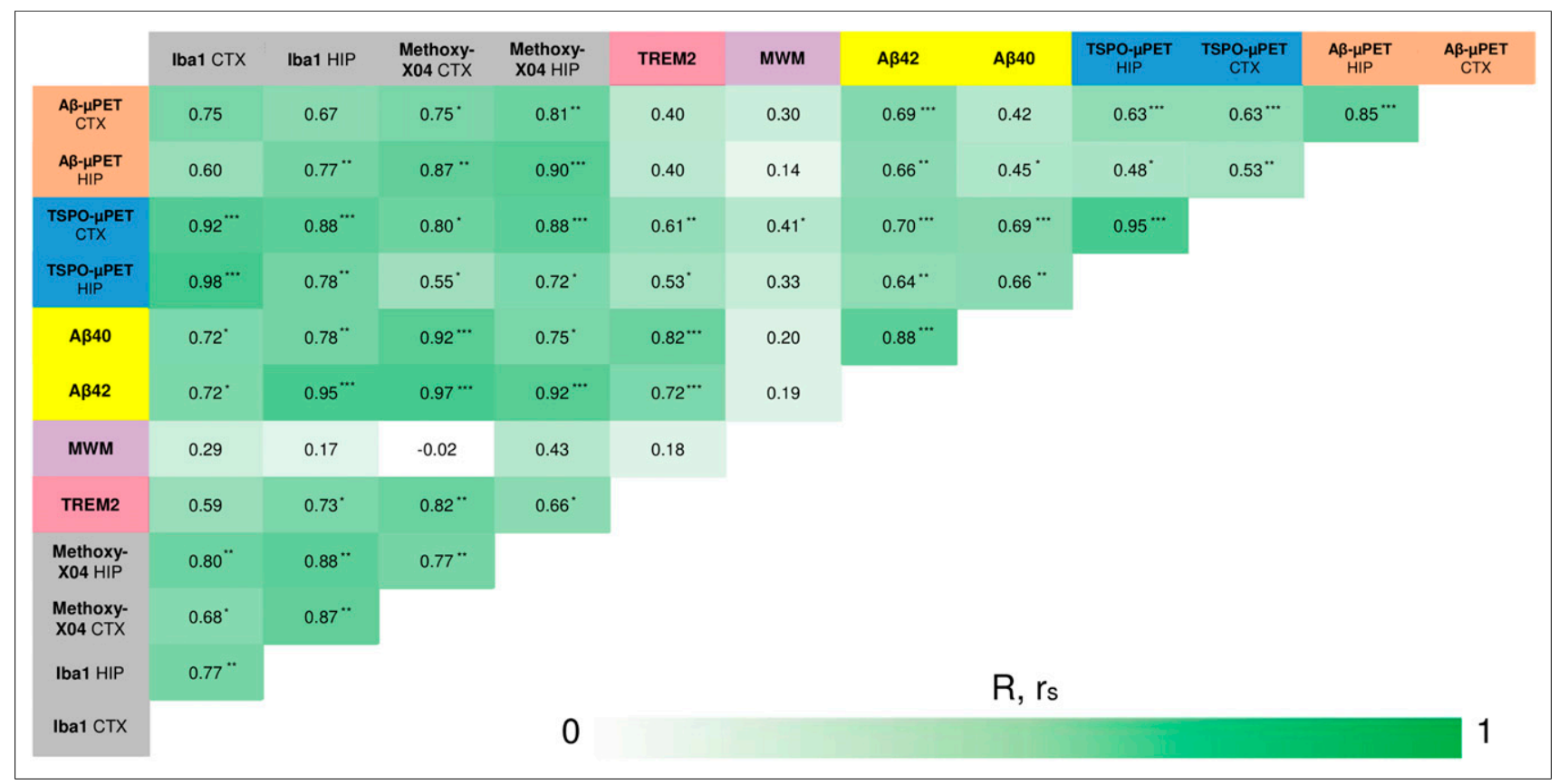

FIGURE 4. Correlation analyses of all terminal readouts. Pearson coefficients of correlation (R) were calculated for normally distributed readouts (small-animal PET, behavior, Iba1, methoxy-X04). For remaining not normally distributed readouts, Spearman coefficients of correlation ( $\left.r_{s}\right)$ were calculated. ${ }^{\star} P<0.05$. ${ }^{\star \star} P<0.01$. ${ }^{\star \star \star} P<0.001$. CTX $=$ cortex; HIP $=$ hippocampal; MWM $=$ Morris Water Maze; TREM2 $=$ Triggering receptor expressed on myeloid cells 2 . 
present $A p p^{N L-G-F}$ model mice, which are vulnerable to more stress-related drop-outs than are other amyloid mouse models (10). Although full kinetic modeling with arterial blood sampling represents the gold standard for small-animal PET quantification, that approach is hardly feasible in mouse studies encompassing up to 4 pairs of PET sessions. Therefore, we used a variance analysis for both PET tracers together with immunohistochemistry assessment to identify the most valid pseudo reference tissue, which proved to be PAG of the mesencephalon. Validation in serial dual PET imaging revealed robust quantification of SUVR relative to PAG, and terminal assessments substantiated our use of this pseudo reference tissue through the excellent correlation of terminal PET results with immunohistochemistry gold standards. A low dropout rate during serial PET imaging $(<10 \%$ per time point) also encourages the use of our newly established SUVR protocol. We note that the SUVR range fell below unity for quantification of both tracers, because of higher unspecific binding in the PAG reference tissue than in cortical or hippocampal target regions. Using the reference tissue normalization but reducing variance in the population stabilized PET quantification, just as in our previous investigations of both ligands $(16,24)$.

Another aspect of the present model concerns the fraction of dense fibrillar $\mathrm{A} \beta$ in the plaques of $A p p^{N L-G-F}$ mice, which is lower than in other transgenic amyloid mouse models. This is an important technicality, as fluorinated A $\beta$ PET tracers such as ${ }^{18} \mathrm{~F}$-florbetaben have high affinity for dense fibrillary plaques but exhibit only low binding in diffuse plaques (25). As expected from this fact, we observed a lesser longitudinal increase for ${ }^{18} \mathrm{~F}$-florbetaben binding than for ${ }^{18} \mathrm{~F}-\mathrm{GE}-180$ from the plaque onset until the full-blown pathology occurring at 10 mo of age (9.1\% vs. $19.8 \%)$. In contrast, we had earlier found similar increases of the same 2 radioligands in APP-SL70 (18.3\% vs. $17.6 \%)(26)$ and PS2APP mice (+19.8\% vs. $+20.2 \%$ ) (16). Thus, whereas quantitative $\mathrm{A} \beta$ imaging to ${ }^{18} \mathrm{~F}$-florbetaben PET is feasible in $A p p^{N L-G-F}$ mice, the tracer misses at least half of the true plaque burden, which constitutes a weakness of using this particular radioligand in the knock-in mouse model. This property needs to be addressed in future studies of $A \beta$-targeting therapies or genetic modifications with differential effects on the expressions of dense and diffuse parts of the plaque (27).

Voxelwise group contrasts between knock-in and wild-type animals revealed increased ${ }^{18} \mathrm{~F}$-florbetaben uptake in homozygous $A p p^{N L-G-F}$ mice already at 5 mo of age. Notably, early increases in amyloid binding were present in the thalamus of homozygous and heterozygous $A p p^{N L-G-F}$ animals. This finding in the thalamus of knock-in mice is in contrast to sporadic $\mathrm{AD}$, and the thalamus is not typically considered a target region for therapy studies in AD mouse models.

Serial PET analyses and terminal assessments of our study indicated parallel increases of amyloidosis and microglial activation with age in the transgenic knock-in mice. The observed strong correlations between cortical TSPO and A $\beta$ readouts were expected from the results of a published study, which demonstrated a link between amyloidosis and neuroinflammation based on comparative profiling of cortical gene expression in AD patients and in the $A p p^{N L-G-F}$ mouse model (13). Our recent study of PS2APP mice showed that the concentration of sTrem2, which is expressed by microglia as a mediator of phagocytic clearance of debris $(6)$, strongly correlates with TSPO and A $\beta$ PET signals (28). The present biochemical analysis of sTrem 2 also showed strong correlations with terminal TSPO PET but not with A $\beta$ PET. This finding may indicate that sTrem 2 serves as a valid biomarker for microglial activation in $A p p^{N L-G-F}$ mice but that its expression is not so tightly coupled to fibrillar A $\beta$ levels in $A p p^{N L-G-F}$ mice as in PS2APP mice.

Recent publications report that homozygous $A p p^{N L-G-F}$ mice show a subtle, progressive deterioration in performance of spatial learning trials, deficits in flexible learning, and reduced attentional performance compared with wild-type (10). Consistent with these findings, we observed a significant deficit in spatial learning in homozygous $A p p^{N L-G-F}$ mice in performing the hippocampus-related Morris water maze test. However, the learning and memory deficits in $A p p^{N L-G-F}$ mice should be further investigated since another study has reported intact learning and memory in homozygous $A p p^{N L-G-F}$ mice at the age of as much as 15-18 mo (12). Spatial learning performance at 10 mo of age did not correlate with longitudinal A $\beta$ PET or with terminal immunohistochemistry or biochemical measures of amyloidosis, as is in line with a recent review of different transgenic mouse models of $\mathrm{AD}$ (29). Our previous study with TSPO PET in PS2APP revealed some evidence for an association between consistently strong early and terminal neuroinflammation with a better preservation of cognitive function (30), suggesting a net protective effect of microglial activation. In contrast, the deterioration in spatial learning in aged $A p p^{N L-G-F}$ mice correlated significantly with increased cortical TSPO PET SUVR at the terminal time point. With regard to the specific plaque composition observed in $A p p^{N L-G-F}$, which has less dense but more diffuse plaques than the first-generation amyloid mouse models, the present findings call for further examination of the specific role of microglial activation in $A p p^{N L-G-F}$ neuropathology. Furthermore, we should in future consider applying other behavioral assessments in addition to the Morris water maze test of spatial learning. Intermouse-model comparisons of findings from imaging in conjunction with other biomarkers are summarized in Supplemental Table 2.

Molecular imaging with PET uniquely affords longitudinal monitoring of disease-related alterations and interventions in individual animals and can allow prediction of progression and therapeutic effects from early baseline characteristics $(14,15)$. Recent therapeutic studies on transgenic mouse models monitored by PET, such as using an inhibitor of the $\beta$-site APP-cleaving enzyme 1 , have already shown encouraging results with respect to delayed pathology $(14,31,32)$. Our serial in vivo PET results together with ex vivo observations in $A p p^{N L-G-F}$ mice, representing an aggressively neurotoxic knock-in amyloid model with cognitive impairment, support the use of these methods for interventional studies, especially when fibrillary parts of the plaque are targeted by the therapy, as is especially relevant for antiamyloid antibodies.

\section{CONCLUSION}

Analysis of $\mathrm{A} \beta$ and TSPO PET imaging in $A p p^{N L-G-F}$ mice is complicated by the widespread cerebral pathology and relatively low fibrillarity of $A \beta$ plaques but is feasible using PAG as a pseudo reference region. Progression of neuropathology can be tracked by serial ${ }^{18} \mathrm{~F}$-florbetaben and ${ }^{18} \mathrm{~F}-\mathrm{GE}-180$ PET in homozygous $A p p^{N L-G-F}$ mice, whereas heterozygous $A p p^{N L-G-F}$ animals present only minor changes to these methods. The combination of PET with a test of cognition in this new knock-in AD model, $A p p^{N L-G-F}$, is a promising test-bed for preclinical drug development.

\section{DISCLOSURE}

Florbetaben precursor was provided by Life Molecular. GE Healthcare made GE-180 cassettes available through an early-access 
model. Seed funding was provided by Verein zur Förderung von Wissenschaft und Forschung an der Medizinischen Fakultät der Ludwig-Maximilians-Universität München. This work was supported by the Deutsche Forschungsgemeinschaft (DFG) by a grant to Matthias Brendel and Axel Rominger (BR4580/1-1 and RO5194/1-1) and within the framework of the Munich Cluster for Systems Neurology (EXC1010SyNergy). Peter Bartenstein and Axel Rominger received speaking honoraria from Life Molecular; Irena Brzak and Ulf Neumann are employees of Novartis. No other potential conflict of interest relevant to this article was reported.

\section{ACKNOWLEDGMENTS}

We thank Karin Bormann-Giglmaier for excellent technical assistance. We acknowledge Inglewood Biomedical Editing for manuscript editing.

\section{KEY POINTS}

QUESTION: Is it possible to monitor preclinical trials using APP knock-in mice by means of small-animal PET for $A \beta$ and $18 \mathrm{kDa}$ TSPO?

PERTINENT FINDINGS: This longitudinal preclinical investigation revealed progressively increasing uptake of PET tracers for $A \beta$ and $18 \mathrm{kDa}$ TSPO in APP knock-in mice. Terminal PET findings were highly correlated with ex vivo gold standard assessments.

IMPLICATIONS FOR PATIENT CARE: PET in APP knock-in mice present a new instrument for bench to bedside therapy monitoring without interference from APP overexpression.

\section{REFERENCES}

1. Ziegler-Graham K, Brookmeyer R, Johnson E, Arrighi HM. Worldwide variation in the doubling time of Alzheimer's disease incidence rates. Alzheimers Dement. 2008;4:316-323.

2. Braak H, Braak E. Demonstration of amyloid deposits and neurofibrillary changes in whole brain sections. Brain Pathol. 1991;1:213-216.

3. Hyman BT, Phelps CH, Beach TG, et al. National Institute on Aging-Alzheimer's Association guidelines for the neuropathologic assessment of Alzheimer's disease. Alzheimers Dement. 2012;8:1-13.

4. Serrano-Pozo A, Frosch MP, Masliah E, Hyman BT. Neuropathological alterations in Alzheimer disease. Cold Spring Harb Perspect Med. 2011;1:a006189.

5. Querfurth HW, LaFerla FM. Alzheimer's disease. N Engl J Med. 2010;362:329344 .

6. Heneka MT, Carson MJ, Khoury JE, et al. Neuroinflammation in Alzheimer's disease. Lancet Neurol. 2015;14:388-405.

7. Jonsson T, Atwal JK, Steinberg S, et al. A mutation in APP protects against Alzheimer's disease and age-related cognitive decline. Nature. 2012;488:96-99.

8. Hsiao K, Chapman P, Nilsen S, et al. Correlative memory deficits, $A \beta$ elevation, and amyloid plaques in transgenic mice. Science. 1996;274:99-102

9. Sasaguri H, Nilsson P, Hashimoto S, et al. APP mouse models for Alzheimer's disease preclinical studies. EMBO J. 2017;36:2473-2487.

10. Masuda A, Kobayashi Y, Kogo N, Saito T, Saido TC, Itohara S. Cognitive deficits in single App knock-in mouse models. Neurobiol Learn Mem. 2016;135:73-82.
11. Saito T, Matsuba Y, Mihira N, et al. Single App knock-in mouse models of Alzheimer's disease. Nat Neurosci. 2014;17:661-663.

12. Sakakibara Y, Sekiya M, Saito T, Saido TC, Iijima KM. Cognitive and emotional alterations in App knock-in mouse models of $\mathrm{A} \beta$ amyloidosis. BMC Neurosci. 2018;19:46.

13. Castillo E, Leon J, Mazzei G, et al. Comparative profiling of cortical gene expression in Alzheimer's disease patients and mouse models demonstrates a link between amyloidosis and neuroinflammation. Sci Rep. 2017;7:17762.

14. Brendel M, Jaworska A, Overhoff F, et al. Efficacy of chronic BACE1 inhibition in PS2APP mice depends on the regional A $\beta$ deposition rate and plaque burden at treatment initiation. Theranostics. 2018;8:4957-4968.

15. Brendel M, Jaworska A, Herms J, et al. Amyloid-PET predicts inhibition of de novo plaque formation upon chronic $\gamma$-secretase modulator treatment. Mol Psychiatry. 2015;20:1179-1187.

16. Brendel M, Probst F, Jaworska A, et al. Glial activation and glucose metabolism in a transgenic amyloid mouse model: a triple-tracer PET study. J Nucl Med. 2016;57:954-960.

17. Brendel M, Focke C, Blume T, et al. Time courses of cortical glucose metabolism and microglial activity across the life span of wild-type mice: a PET study. J Nucl Med. 2017;58:1984-1990.

18. Deussing M, Blume T, Vomacka L, et al. Coupling between physiological TSPO expression in brain and myocardium allows stabilization of late-phase cerebral $\left[{ }^{18}\right.$ F $]$ GE180 PET quantification. Neuroimage. 2018;165:83-91.

19. Rominger A, Brendel M, Burgold S, et al. Longitudinal assessment of cerebral b-amyloid deposition in mice overexpressing Swedish mutant b-amyloid precursor protein using ${ }^{18}$ F-florbetaben PET. J Nucl Med. 2013;54:1127-1134.

20. Sawiak SJ, Wood NI, Williams GB, Morton AJ, Carpenter TA. Voxel-based morphometry in the R6/2 transgenic mouse reveals differences between genotypes not seen with manual 2D morphometry. Neurobiol Dis. 2009;33:20-27.

21. Bromley-Brits K, Deng Y, Song W. Morris water maze test for learning and memory deficits in Alzheimer's disease model mice. J Vis Exp. 2011;53:e2920

22. Dorostkar MM, Dreosti E, Odermatt B, Lagnado L. Computational processing of optical measurements of neuronal and synaptic activity in networks. J Neurosci Methods. 2010;188:141-150.

23. Neumann U, Rueeger H, Machauer R, et al. A novel BACE inhibitor NB-360 shows a superior pharmacological profile and robust reduction of amyloid-beta and neuroinflammation in APP transgenic mice. Mol Neurodegener. 2015;10:44.

24. Overhoff F, Brendel M, Jaworska A, et al. Automated spatial brain normalization and hindbrain white matter reference tissue give improved $\left[{ }^{18} \mathrm{~F}\right]$-florbetaben PET quantitation in Alzheimer's model mice. Front Neurosci. 2016;10:45.

25. Catafau AM, Bullich S, Seibyl JP, et al. Cerebellar amyloid-beta plaques: how frequent are they, and do they influence ${ }^{18} \mathrm{~F}$-florbetaben SUV ratios? J Nucl Med. 2016;57:1740-1745.

26. Blume T, Focke C, Peters F, et al. Microglial response to increasing amyloid load saturates with aging: a longitudinal dual tracer in vivo muPET-study. J Neuroinflammation. 2018;15:307.

27. Ulrich JD, Ulland TK, Mahan TE, et al. ApoE facilitates the microglial response to amyloid plaque pathology. $J$ Exp Med. 2018;215:1047-1058.

28. Brendel M, Kleinberger G, Probst F, et al. Increase of TREM2 during aging of an Alzheimer's disease mouse model is paralleled by microglial activation and amyloidosis. Front Aging Neurosci. 2017;9:8.

29. Foley AM, Ammar ZM, Lee RH, Mitchell CS. Systematic review of the relationship between amyloid-beta levels and measures of transgenic mouse cognitive deficit in Alzheimer's disease. J Alzheimers Dis. 2015;44:787-795.

30. Focke C, Blume T, Zott B, et al. Early and longitudinal microglial activation but not amyloid accumulation predict cognitive outcome in PS2APP mice. $\mathrm{J} \mathrm{Nucl}$ Med. 2019;60:548-554.

31. Deleye S, Waldron AM, Verhaeghe J, et al. Evaluation of small-animal PET outcome measures to detect disease modification induced by BACE inhibition in a transgenic mouse model of Alzheimer disease. J Nucl Med. 2017;58:1977-1983.

32. Meier SR, Syvanen S, Hultqvist G, et al. Antibody-based in vivo PET imaging detects amyloid-beta reduction in Alzheimer transgenic mice after BACE-1 inhibition. J Nucl Med. 2018;59:1885-1891. 